\title{
Excerpts of Flucht and Zuflucht:Erinnerunger an eine bewegte Jugend, by Josef Eisinger.
}

Translated from German into English and French by Marie-Catherine Allard 
In the period between the Anschluss and the beginning of WWII [...] so-called Kindertransports had been proposed to Eichmann by a remarkably courageous Dutch woman, Gertrud Wijsmuller-Meijer. After suffering many indignities and arrest, her scheme succeeded in saving the lives of thousands of children. To the best of my knowledge, England was the only country willing to accept these children and it was by means of such Kindertransport that I made my escape from Vienna in April 1939. ${ }^{6}$

I can only surmise how my name found its way onto a Kindertransport list of children scheduled to leave for England. Each child had to be sponsored either by an organization or an individual, and it is likely that Mr. deCosta had lent his name as my sponsor. Each child was allowed to take one small suitcase, and I recall lengthy discussion regarding the selection of items that would be most useful to me in the Great Unknown. [...]

When the day of my departure arrived, Mutti
In der Zeit zwischen dem Anschluss und dem Beginn des Zweiten Weltkriegs [...] sogenannten Kindertransporte wurden Eichmann von einer außergewöhnlich tapferen Holländerin, Geertruida Wijsmuller-Meijer, vorgeschlagen. Nach demütigenden Verhandlungen mit Eichmann gelang es ihr, zehntausend Kindern das Leben zu retten. Auch ich entkam den Nazis im März 1939 mit einem solchen Kindertransport. ${ }^{15}$

Ich kann nur vermuten, wie mein Name auf die Liste der Kinder für den Kindertransport nach England gekommen ist. Jedes Kind musste entweder von einer Organisation oder von einer Person gesponsert werden, wahrscheinlich war Herr de Costa mein Sponsor. Jedes Kind durfte einen kleinen Koffer mitnehmen, und ich erinnere mich an lange Diskussionen, welche Gegenstände in der unbekannten Ferne am nützlichsten sein würden. [...]
Au cours de la période entre l'Anschluss et le début de la Seconde Guerre mondiale [...] des transports appelés Kindertransport furent proposés à Eichmann par Gertrud Wijsmuller-Meijer, une Néerlandaise remarquablement courageuse. Après un nombre de négociations et d'arrestations humiliantes, elle réussit à mettre son plan à exécution et sauva ainsi la vie de milliers d'enfants. À ma connaissance, l'Angleterre fut le seul pays à accepter les Kinder. C'est à bord de l'un de ces Kindertransports qu'en avril I939 je pus mévader de Vienne. ${ }^{6]}$

Je ne peux que supposer la façon dont mon nom s'est retrouvé sur l'une des listes d'enfants devant partir à bord du Kindertransport pour l'Angleterre. Chaque enfant devait être parrainé soit par une organisation où un individu, et il est fort probable que Monsieur deCosta accepta de me parrainer pour la forme. Chaque enfant était autorisé à apporter une petite valise; je me souviens des longues discussions au sujet du choix des articles qui me seraient les plus 
accompanied me to the railway station, the Westbahnhof. I wore my tweed knickerbockers, the usual attire of Viennese teenager (I had just turned fifteen), an outfit that prompted people in England to ask if I was on my way to play golf. The station was crawling with police and Gestapo men, who searched our suitcases for money and other contraband. They taunted the unhappy parents and forbade them to display any emotion or distress while saying good-bye on the platform-on pain of the transport being cancelled.

${ }^{6}$ The Kindertransport story is told in "And the Policeman Smiled" by Barry Turner (ISBN o 74750620 5); it has also been told in the words of many Kinder, including this one, in "I Came Alone, The Stories of the Kindertransports" by B. Leverton and S. Lowensohn, eds. (ISBN o 86332566 I).

Eisinger 40-4I, 56 (Original)
Am Tag meiner Abfahrt begleitete mich Mutti zum Westbahnhof. Ich trug Knickerbocker, was für Wiener Teenager (ich war gerade 15 geworden) üblich war, was allerdings Engländer veranlasste, mich zu fragen, ob ich Golf spielen ginge. Der Bahnhof wimmelte von Polizei und Gestapo, die unsere Koffer nach Geld und Schmuggelgut durchsuchten. Sie verspotteten die unglücklichen Eltern und verboten ihnen, Gefühle beim Abschied am Bahnsteig zu zeigen - ansonsten würde der Transport abgesagt.

\section{${ }^{15}$ Die Geschichte der}

Kindertransporte wird in And the Policeman Smiled von Barry Turner dargestellt (I990); sie wurde auch mit den Worten vieler Kinder erzählt, unter anderem in I Came Alone, The Stories of the Kindertransports von B. Leverton und S. Lowensohn (1990).

\section{DOEW 72-73}

utiles au cours de ce grand voyage vers l'inconnu. [...]

Lorsque le jour de mon départ arriva, Mutti m'accompagna à la gare de l'ouest. Je portais mon pantalon en tweed de style knickerbockers, ce qui était la tenue habituelle d'un adolescent viennois (je venais d'avoir quinze ans). Cette tenue incita les Anglais à me demander si jétais en route pour le golf. La gare grouillait de policiers et d'hommes de la Gestapo qui fouillèrent nos valises à la recherche d'argent et d'objets de contrebande. Ils se moquèrent des malheureux parents et leur interdirent d'afficher quelques signes démotions ou de détresse au moment de dire au revoir sur la plateforme, sous peine d'annuler le transport.

${ }^{6}$ L'histoire du Kindertransport est décrite par Barry Turner dans And the Policeman Smiled (ISBN o 7475 0620 5); elle fut aussi racontée par les Kinder, dont je fais parti, dans I Came Alone, The Stories of the Kindertransports édité par B. Leverton et S. Lowensohn. (ISBN o 86332566 I). 


\section{Lubes Taueluch!}

Sch farsite den

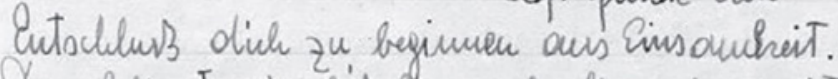
Das hlingt inc bischen geschoshlen abor es int or. Ide will, doin Du viri in wer ine guter Fremol bist, in Tisster in Monrigen Ithuden. Jich hate Dieh gerne and Dime Ouffoche wirol es sein alle reime Crimer ungun rud

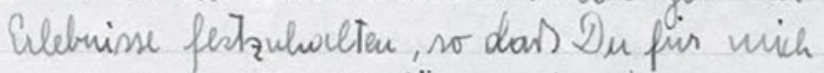
wert voller wist als fïr andere Mens hen, dene ich haufte Dieh mu 3d. his Woohooth. Jin hoffe, dows

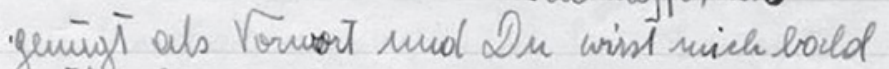
mähir benuen lerven. 
[22 Oct. I939]

Dear Diary!

I decided to begin keeping you out of loneliness. That sounds a bit puffed up, but that's the way it is. I hope you will be a good friend to me that consoles me during sad hours. I am fond of you and it will be your task to hang on to all my memories and adventures, so that you will become more valuable to me than anyone else, for I acquired you at Woolworth's for $3 d$. I hope this will do as an introduction and that you will soon get to know me better.

(In the English version, this entry continues with the next entry: "I am at the present." However, in the original German version, both entries are distinct)

Eisinger 52 (translated by himself from his German entry into English)
Liebes Tagebuch! Ich faßte den Entschlu $\beta$ dich zu beginnen aus Einsamkeit. Das klingt ein bißchen geschwollen aber es ist so. Ich will, da $\beta$ Du mir immer ein guter Freund bist, ein Tröster in traurigen Stunden. Ich habe Dich gerne und Deine Aufgabe wird es sein alle meine Erinnerungen und Erlebnisse festzuhalten, so da $\beta$ Du für mich wertvoller wirst als für andere Menschen, denn ich kaufte Dich um 3 d. bei Woolworth. Ich hof$f e$, das genügt als Vorwort und Du wirst mich bald näher kennen lernen.

DOEW 87 (Original)
Cher Journal!

Ma solitude m'a poussé à prendre la décision de t'écrire. Ça peut sembler un peu exagéré, mais c'est comme ça. Je souhaite que tu sois toujours un bon ami pour moi et que tu me consoles lors de périodes difficiles. Je t'aime déjà beaucoup et ta tâche sera de conserver tous mes souvenirs et expériences afin que tu deviennes plus précieux que quiconque à mes yeux; je t'ai acheté pour 3 sous chez Woolworth. J'espère que cela suffit comme introduction et que tu apprendras bientôt à mieux me connaître.

Ma traduction 


\section{Bishon Morhtor, $22 \cdot \underline{x} \cdot 1939$. \\ Selu bin noment ocu in eirem}

jönmerlichen belicuen Dorf in England mad arbite

als "lad" bei envem biedecen Yorkshire-Formen

mitt Nanen Mr. Morland.

Dus ist in Kinzze nueive jutzige

Itellung. Wie ich qu dieser It ellung ham

nud worrun ich Deutsch ollreibe werole ich Din

jitz' erhloiren.

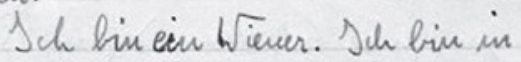

Vien gebren, lin in Wien in die Ichule gegonugen,

ich habe in Wien minee Vindheit erlebt, ich

hin in Wien glichlich nud myelichlich yeresen.

Sch bin thene sin wiever. Se bm solonge

in diser Itodat yevesen bis mich ein thert onf-

geforolert hout sie zu verlarsan. Es wou tlen titter.

Jih woluite in III. Bezinh Risuersth.

29) Tirr 1. Is int eive sclioves thour nud ich

habe die essten 14 Jahre meines debens doriu

DOEW p.93

I am at the present in a miserable little village in England and work as a 'lad' for a humble farmer, $\mathrm{Mr}$. Morland by name. That, in a nutshell is my present position and I will now explain how I got here and why I am writing in German.

I am a Viennese. I was born in Vienna, went to school in Vienna, experienced my childhood in Vienna, was happy and unhappy in
[Bishop Monkton, 22.

Oktober 1939]

Ich bin momentan in einem jämmerlichen kleinen Dorf in England und arbeite als "lad" bei einem biederen Yorkshire Farmer mit Namen Mr. Morland. Das ist in Kürze meine jetzige Stellung. Wie ich zu dieser Stellung kam und warum ich Deutsch schreibe werde ich Dir jetzt erklären.
Bishop Monkton, 22 octobre I939

Je suis présentement dans un misérable petit village d'Angleterre et je travaille comme 'lad' chez un modeste fermier du Yorkshire nommé $M$. Morland. Ceci est en résumé ma situation actuelle. Je vais maintenant t'expliquer comment j'en suis arrivé à ce point et pourquoi je t'écris en allemand. 
Vienna, in short I am a

Viennese.

After describing where I lived and where I went to school, I listed the members of my family [.]

Eisinger $p .52$
Ich bin ein Wiener. Ich bin in Wien geboren, bin in Wien in die Schule gegangen, ich habe in Wien meine Kindheit erlebt, ich bin in Wien glücklich und unglücklich gewesen. Ich bin eben ein Wiener. Ich bin solange in dieser Stadt gewesen bis mich ein Herr aufgefordert hat sie zu erlassen. Es war Herr Hitler.

Ich wohnte im III. Bezirk,

Reisnerstr. 29 Tür I. Es

ist ein schönes Haus und ich habe die ersten I4 Jahre meines Lebens darin verbracht.

Die nächsten Eintragungen beschreiben mein Familienleben in herzlichen Worten [.]

DOEW p. 87 (Original is in German. Underlined are the words that were not included by Eisinger in his English Translation).
Je suis Viennois. Je suis né à Vienne, suis allé à l'école à Vienne, j'ai vécu mon enfance à Vienne, j'étais heureux et malheureux à Vienne. Bref, je suis viennois. Je suis resté dans cette ville jusqu'à ce qu'un homme insiste pour que je la quitte. Cet homme était Hitler.

Je demeurais dans le troisième arrondissement, au 29 Reisnerstraße, porte numéro I. C'est une belle maison et j'y ai vécu les I4 premières années de ma vie.

Après avoir décrit où j'habitais et où j'allais à l'école, j'ai énuméré le nom des membres de ma famille.

Ma traduction 


The destination of these
transports was never offi-
cially announced and one
of the transports actually
sailed to Australia.
[...]

When I came on deck early one morning I had my first glimpse of the American continent. $[\ldots]$

Once ashore, we were subjected to yet another thorough search of our meager possessions, this time by our Canadian guards who relieved us of any remaining valuables. A train awaited us on a siding near the dock, and after a journey of a few hours, we arrived at the town of Trois-Rivieres. From the railway station we were marched through streets lined with curious and sometimes hostile crowds of French-Canadians who had expected fierce Nazi paratroopers, as additional inmates of the POW camp housed in the town's exhibition grounds. They must have been taken aback by our motley crew of civilians, including a contingent of bearded and somberly dressed orthodox Jews; were they
Ich nenne es ein

Gerücht, da das Ziel der Transporte niemals offiziell bekanntgegeben wurde - einer der Transporte segelte tatsächlich nach Australien. ${ }^{22}$

[...]

Als ich eines frühen Morgens an Deck kam, blickte ich zum ersten Mal auf den amerikanischen Kontinent.

[...]

Unsere Besitztümer wurden erneut gründlich durchsucht, diesmal durch kanadische Wachsoldaten, die uns jegliche verbliebenen Wertgegenstände abnahmen. Nahe dem Hafen wartete ein Zug auf uns und wir kamen nach einer mehrstündigen Fahrt in der Stadt Trois-Rivières an. Vom Bahnhof wurden wir durch eine Straße geführt, die mit neugierigen und manchmal auch feindseligen Franko-Kanadierinnen und -Kanadiern gesäumt waren. Sie hatten grimmige $\mathrm{Na}-$ zi-Fallschirmjäger für das Kriegsgefangenenlager auf dem Ausstel-
La destination de ces transports ne fut jamais officiellement annoncée-l'un de ces transports navigua finalement vers l'Australie. ${ }^{5}$ [...]

Au moment où j'arrivai sur le pont tôt un matin, j'aperçus pour la première fois le continent américain.

[...]

Une fois sur la terre ferme, nous fûmes soumis encore une fois à une fouille approfondie de nos maigres possessions, cette fois cependant, par nos gardes canadiens qui saisirent ce qu'il nous restait de valeur. Un train nous attendait près du quai, et après un trajet de quelques heures, nous arrivâmes dans la ville de Trois-Rivières. De la gare, nous marchâmes à travers les rues bordées de Canadiens français curieux et parfois hostiles qui eux s'attendaient que les nouveaux détenus du camp de prisonniers de guerre, situé au cœur du Parc de l'Exposition de la ville, soient d'inquiétants parachutistes nazis. Ils durent être surpris par la diversité de notre groupe 
cleverly disguised Fifth Columnists?

${ }^{5}$ Four 'prison ships' carried a total of 6,675 internees and prisoners-of-war to Canada. One, the Arandora Star, was sunk by a U-boat, with the loss of I,Ooo lives. A fifth ship, the Dunera, transported the Arandora Star survivors and others to camps in Australia. She had a narrow escape when a torpedo glanced off her bow. (Koch, op. cit.).

Eisinger 69, 71, 84 lungsgelände der Stadt erwartet und mussten daher von der bunten Mischung unseres zivilen Aufmarsches, darunter bärtige, dunkel gekleidete orthodoxe Juden, überrascht gewesen sein: War das die listig verkleidete „Fünfte Kolonne"?

${ }^{22}$ Vier "Gefängnisschiffe" brachten insgesamt 6.675 Internierte und Kriegsgefangene nach Kanada. Eines, die Arandora Star, wurde durch ein deutsches U-Boot versenkt, I.ooo Menschen verloren dabei ihr Leben. Ein fünftes Schiff, die Dunera, transportierte die Überlebenden der Arandora Star und andere in Lager in Australien. Auch diese entkamen knapp, als ein Torpedo haarscharf den Bug verfehlte. (Siehe Koch, Deemed Suspect) composé de civils, incluant un contingent de juifs orthodoxes barbus et sombrement vêtus; était-ce les membres de la «Cinquième Colonne» habilement déguisés?

${ }^{5}$ Quatre bateaux-prisons transportèrent un total de 6675 internés civils et prisonniers de guerre au Canada. L'un d'eux, l'Arandora Star, fut coulé par un sous-marin allemand. I ooo passagers perdirent la vie. Un cinquième navire, le Dunera, transporta les survivants du naufrage, et d'autres prisonniers, vers des camps de prisonniers australiens. Le Dunera échappa de justesse à l'attaque d'une torpille. (Voir Koch, Eric. Deemed Suspect: A Wartime Blunder)

Ma traduction 
It soon became clear that Britain had merely requested the Canadian government to keep their prisoners for safekeeping, 'for the duration', without differentiating between POWs and civilian internees. It was to take a long time to convince the Canadian authorities that almost all of their civilian charges were, in fact, Jewish or political refugees and were passionately opposed to the Nazis.

The camp was already inhabited by genuine German POWs and as we marched into the camp, they welcomed us by serving a meal of hard-boiled eggs and raw bacon. The camp, was, as usual, unprepared for our arrival. We were taken to an empty exhibition hall where we slept on straw pallets on the cement floor and where immediately a long line formed in front of the only working toilet.

We encountered other unheard of amenities, such as showers and a recreation ground were soccer games and other sporting events were quickly organized. But
Bald wurde evident, dass die Briten die kanadische Regierung nur ersucht hatten, auf ihre deutschen Gefangenen aufzupassen, ohne zwischen den Kriegsgefangenen und den Zivilinternierten zu unterscheiden. Es dauerte lange, die kanadischen Behörden zu überzeugen, dass fast alle Zivilisten Juden oder politische Flüchtlinge und vehement gegen die Nazis eingestellt waren.

Im Lager lebten bereits deutsche Kriegsgefangene. Als wir ankamen, wurden wir mit einer Mahlzeit, bestehend aus harten Eiern und rohem Speck, empfangen. Das Lager war, wie üblich, auf unsere Ankunft nicht vorbereitet. Wir wurden in eine leere Ausstellungshalle geführt, wo wir auf Strohsäcken auf dem Zementboden schliefen und wo sich sofort eine lange Schlange vor der einzigen funktionierenden Toilette bildete.

Wir fanden auch andere unglaubliche Annehmlichkeiten vor, wie Dus-
Il est vite devenu clair que la Grande-Bretagne avait seulement demandé au gouvernement canadien de veiller sur ses prisonniers sans faire de distinction entre les prisonniers de guerre et les internés civils. Il fallut beaucoup de temps pour convaincre les autorités canadiennes que presque tous les civils internés étaient, en fait, des réfugiés juifs ou politiques et étaient fortement opposés aux nazis et leurs idéologies.

À notre arrivée au camp, celui-ci était déjà habité par de vrais prisonniers de guerre allemands qui nous accueillirent en nous servant un repas composé d'œufs cuits durs et de bacon cru. Comme d'habitude, le camp n'était pas préparé à notre arrivée.

Nous fûmes emmenés dans une salle d'exposition vide dans laquelle nous dormîmes sur des sacs de paille sur le sol en ciment et où, immédiatement, une longue file se forma devant la seule toilette encore fonctionnelle.

Nous avons découvert 
soon fights broke out between Nazis and anti-Nazis and the guards were obliged to erect a barbed wire barrier to separate the two warring factions.

Eisinger 71 chen und Sportfelder, wo wir rasch Fußballund andere Sportbewerbe organisierten. Aber bald brachen Kämpfe zwischen Nazis und Nazigegnern aus und unsere Wärter, Veteranen aus dem I. Weltkrieg, mussten Zäune errichten, um die zwei Konfliktparteien $\mathrm{zu}$ trennen.

DOEW 107-108 d'autres installations surprenantes, telles que des douches et un terrain sur lequel des parties de soccer et d'autres événements sportifs furent vite organisés. Des bagarres éclatèrent rapidement entre les nazis et les internés antinazis. Nos gardes furent obligés d'ériger une barrière de barbelés pour séparer les deux parties.

Ma traduction

(Camp B, I6 August 1940)

...The train journey was not very pleasant since we were in an ancient carriage. The camp is far from ready and worst of all, there is almost no water. On the day we arrived it rained and we washed in the puddles. In a word, conditions were terrible. You couln't come near the latrine; the smell would knock you down. Now things are much better. We can even work outside the camp. This morning, for instance, I did carpentry, which was a lot of fun. The workmen and the guards are very nice.
[Camp B, I6. August 1940]

Die Bahnreise war nicht sehr angenehm, zumal wir in einem uralten Waggon fuhren. Das Lager ist noch lange nicht fertig, vor allem gibt es fast kein Wasser. Als es am Tage an dem wir ankamen am Abend regnete, wuschen wir uns in den Pfützen. Mit einem Wort es waren schreckliche Umstände. Man konnte sich der Latrine nicht nähern da der Geruch jeden umwarf. Jetzt geht es schon viel besser. Wir können sogar außerhalb des Lagers arbeiten. Heute morgen, zum Beispiel, war ich draußen und habe
Camp B, I6 août 1940

Le voyage en train n'était pas très agréable, surtout que nous avons voyagé à bord d'un vieux wagon. Le camp est loin d'être terminé, surtout qu'il n'y a presque pas d'eau. Comme il a plu le jour de notre arrivée, nous nous sommes lavés dans les flaques d'eau. En un mot, les circonstances étaient terribles. On ne pouvait approcher des latrines puisque l'odeur nous renversait tous.

Maintenant, les choses vont beaucoup mieux. Nous pouvons même travailler en dehors du camp. Ce matin, par exemple, 
Camp B was, in retrospect, a most enriching experience for me. Even though all internees were required to work every day, our simple life left plenty of leisure time for endless discussions and we formed many friendships, including a few lasting lasting [sic] ones.

\section{Eisinger 73}

(The diary entry was originally in German, while the retrospective comment was written in English). getischlert wobei ich mich sehr gut unterhalten habe. Die Arbeiter und die Soldaten sind sehr nett. Auch die Kost scheint jetzt besser zu werden, nachdem wir in der ersten Zeit kaum etwas zu essen bekamen.

Das Lager ist ganz von Wald umgeben wie es hier überhaupt unheimlich viel Wald gibt.

Rückblickend war Camp $B$ eine sehr bereichernde Erfahrung für mich. Obwohl die Internierten jeden Tag arbeiten mussten, blieb viel Zeit für endlose Diskussionen und wir schlossen viele Freundschaften, manche für lange Zeit.

DOEW 110

(Original is in German. Underlined are the words that were not included by Eisinger in his English Translation). j'ai fait de la menuiserie, ce qui était très amusant. Les ouvriers et les soldats sont très gentils. Même la nourriture semble s'améliorer maintenant, surtout si on compare au fait qu'au début, nous recevions à peine de quoi manger. Le camp est complètement entouré de forêts puisqu'il y a ici une quantité incroyable de forêts.

Avec du recul, le camp $B$ fut une expérience des plus enrichissantes pour moi. Même si tous les internés devaient travailler tous les jours, notre vie au camp nous laissait beaucoup de temps pour de longues discussions au cours desquels nous avons noué de nombreuses amitiés, dont quelques-unes furent durables. 
(24 August, I940)

The day before yesterday we were issued toilet articles for the first time (towel, razor, shaving brush, toothbrush, etc.)! What's more, uniforms arrived for us, which we refused because they had a huge red circle on the back of the blue jacket and shirt.

\section{Eisinger 75}

[Camp B, 24.-25.

August 1940]

Vorgestern haben wir zum ersten Male Toiletteartikel bekommen (Handtuch, Rasierapparat, Pinsel, Zahnbürste usw.). Ausserdem kamen Uniformen für uns alle, die wir jedoch nicht annahmen da sie am Rücken riesige rote runde Flecken hatten.

\section{DOEW 113}

Original $=$ Deutsch
Camp B, 24-25 août 1940

Avant-hier, nous avons reçu, pour la première fois, des articles de toilette (une serviette, un rasoir et un blaireau, une brosse à dents, etc.). Des uniformes nous ont aussi été apportés, mais nous avons refusé de les mettre puisqu'ils ont un énorme cercle rouge dans le dos de la veste et de la chemise bleues.

Ma traduction
The discussions and the confrontations with the military regarding our POW status continued in Camp A, where we moved sometime during the summer of 194I. Since this camp was within the town of Farnham and not utterly isolated like Camp B, we mounted a large sign proclaiming our anti-Nazi sentiments on the roof of a hut, which was visible from beyond the wire. We also continued to object to having to wear the prison uniforms and we refused to use POW stationary since it was handled by the
Die Diskussionen und Konfrontationen mit den Militärbehörden bezüglich unseres Status als Kriegsgefangene dauerten im Camp A, wohin wir im Sommer 194I verlegt wurden, an. Da dieses Lager innerhalb der Stadt Farnham lag und nicht wie Camp B völlig isoliert war, montierten wir ein großes Plakat auf das Dach einer unserer Hütten, das auch jenseits des Stacheldrahts sichtbar war und unsere Gegnerschaft zum Nationalsozialismus ausdrückte. Wir wehrten uns auch noch immer gegen die
Les discussions ainsi que les confrontations avec les autorités militaires concernant notre statut de prisonniers de guerre se poursuivirent au camp A, l'endroit où nous fûmes transférés au cours de lété 194I. Puisque ce camp était situé dans la ville de Farnham et que, contrairement au camp $B$, il n'était pas tout à fait isolé, nous installâmes une grosse pancarte sur le toit d'une des huttes qui était également visible au-delà des barbelés afin de proclamer notre opposition au nazisme. Nous continuâmes également à 
Swiss Red Cross along with mail from German POWs. We capitulated on the uniforms, since the approaching Canadian winter left us little choice, but won the right to use plain stationery-all issues that loomed as matters of life and death at the time!

Eisinger 77

(Original $=$ English)
Gefängniskleidung und weigerten uns, Briefpapier für Kriegsgefangene $\mathrm{zu}$ verwenden, $\mathrm{da}$ es vom Schweizer Roten Kreuz so wie die Post der deutschen Kriegsgefangenen behandelt wurde. Bei den Uniformen gaben wir angesichts des kommenden kanadischen Winters nach, erreichten aber die Erlaubnis, normales Briefpapier zu verwenden - all das schien in der Zeit von ungeheuer großer Bedeutung.

DOEW 117 nous opposer au fait de devoir porter les uniformes de prisonniers et refusâmes d'utiliser le matériel d'écriture mis à la disposition des prisonniers de guerre parce que leur courrier était traité par la Croix-Rouge suisse, qui elle ne faisait pas la distinction entre les lettres des internés civils et celles des prisonniers de guerre allemands. Bien que nous dussions capituler pour ce qui est des uniformes parce que l'hiver canadien approchait à grands pas et ne nous laissait pas d'autres choix, nous obtînmes le droit d'utiliser du papier ordinaire pour nos lettres-à cette époque, ces détails étaient de vraies questions de vie ou de mort!

Ma traduction
In camp we generally spoke German to each other, which was, after all, our mother tongue, and up to now, I had also kept my diary in German. But beginning with the next entry, I switched to English, crossing a significant
Im Lager sprachen wir meistens Deutsch miteinander, das ja unsere Muttersprache war, und bis dahin hatte ich auch mein Tagebuch auf Deutsch geführt. Aber mit dem folgenden Eintrag wechselte ich zu Englisch und
Au camp, nous nous parlions généralement allemand, cette langue était après tout, notre langue maternelle, et jusqu'à maintenant, j'avais aussi écrit mon journal en allemand. Cependant, l'entrée suivante me fit franchir 
linguistic divide without even noticing it!

$$
\text { (II April, 194I) }
$$

Just now I am spending what I consider the best time I have had during my internment. I am studying all day long and as far as I can judge, am happy doing so... It may seem ridiculous, yet summing up, I must admit that I am glad I got interned.

(I6 July, 194I)

I am now doing physics with Walter and have decided to attend the new calculus course for the matric that Rothberger has started. I work every day making nets and since one needs to work only 2 hours a day I have plenty of time for my studies while earning some money. Our status is said to be about to change, from Monday on we will be allowed to use ordinary stationery.

Eisinger 76-77

(Original diary entries= English) überschritt damit eine bedeutende Sprachgren$z e$, ohne es zu merken.

\section{[II. April 194I]}

Ich verbringe gerade die beste Zeit meiner Internierung. Ich studiere den ganzen Tag, und soweit ich das beurteilen kann, macht es mich glücklich [...] Es mag lächerlich erscheinen, aber alles in allem muss ich zugeben, dass ich froh bin, interniert worden zu sein.

[16. Juli 194I]

Ich lerne nun Physik mit

Walter und habe mich entschlossen, dem neuen

Differentialrechnungskurs für die Matric Prüfung, den Rothberger begonnen hat, beizuwohnen. Ich arbeite jeden Tag Netze zu machen, aber da man nur ungefähr zwei Stunden täglich arbeiten muss, habe ich viel Zeit übrig zu studieren und nebenbei Geld zu verdienen. Unser Status soll sich am Montag ändern, wir werden normales Briefpapier verwenden dürfen.

DOEW 116-117 une importante barrière linguistique: je passai de l'allemand à l'anglais sans même m'en rendre compte.

II avril I94I

Je passe présentement ce que je considère comme le meilleur moment de mon internement. J'étudie toute la journée et pour autant que je sache, ça me rend heureux. Cela peut paraitre ridicule, mais dans l'ensemble, je dois avouer que je suis content d'avoir été interné.

I6 juillet I94I

J'apprends maintenant la physique avec Walter et ai décidé de suivre le nouveau cours de calcul différentiel donné par Rothberger afin de me préparer à l'examen de matricule. Je travaille tous les jours à la confection de moustiquaires et puisqu'il est requis de seulement travailler 2 heures par jour, j'ai beaucoup de temps à consacrer à mes études, et ce, tout en gagnant un peu d'argent. Il parait que notre statut est sur le point de changer et qu'à partir de lundi, nous serons autorisés à utiliser du papier à écrire ordinaire. 
(22 Oct. 194I)

Yesterday I received the most wonderful letter I could receive, namely from Mrs. Mendel promising me and Rappa [Walter Kohn] that they would sponsor us! I was just at the dentist's and George [Sanger] was mixing amalgam when Odizetti [Walter Odze] brought the letter and I nearly fell off the chair.

Ebook 78

Original diary entry $=$ English
[22. Oktober 194I]

Gestern erhielt ich den wunderbarsten Brief, den ich erhalten könnte, nämlich von Frau Mendel, die versprach, mich und Rappa [Walter Kohn] zu sponsern. Ich war gerade beim Zahnarzt und George [Sanger] mischte Amalgam, als Odizetti [Walter Odze] den Brief brachte, und ich fiel fast vom Sessel.

DOEW 120

\section{2 octobre I94I}

Hier, j'ai reçu la plus belle lettre que j'aurais pu recevoir: une dénommée madame Mendel me fit la promesse de nous parrainer Rappa [Walter Kohn] et moi! J'étais chez le dentiste et pendant que George [Sanger] mélangeait l'amalgame dentaire, Odizetti [Walter Odze] apporta la lettre qui faillit me faire tomber de ma chaise.

Ma traduction

Refugees, vio lefs fermany is 1933 and are now Cauadions. Uny huntaud is a Plysintays and Prapiars of the thinaricly of Tormito. Le tame teree couldre s alout yous afe and the are ald copling forseard to teing you tarn.

tith bect Holed fours sincerely Armuo and terthabeudef
Torouts ace $10^{k / 4}$

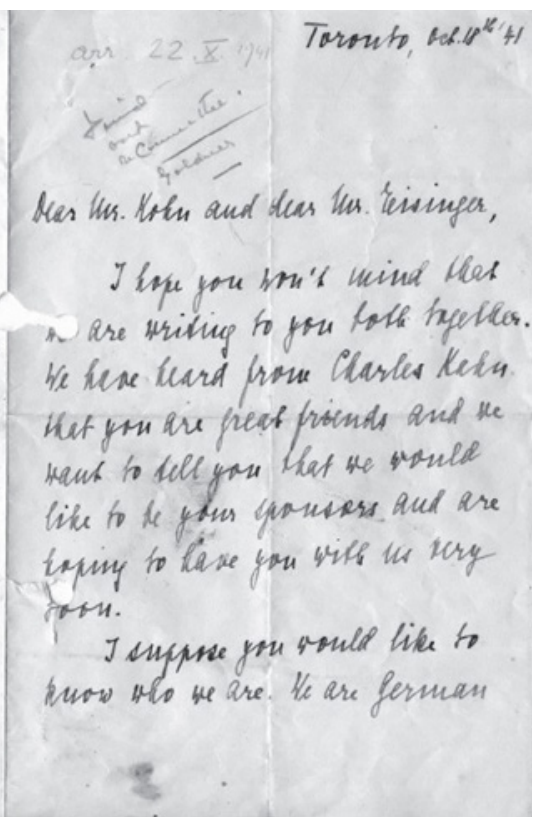


Toronto, Oct. $18^{\text {th }}$ " $4 \mathrm{I}$

Arrived 22 X 194I

Dear Mr. Kohn and dear

Mr. Eisinger,

I hope you won't mind that we are writing to you both together. We have heard from Charles Kohn that you are great friends and we want to tell you that we would like to be your sponsors and are hoping to have you with us very soon.

I suppose you will like to know who we are. We are German Refugees, who left Germany in 1922 and are now Canadians. My husband is a Physiologist and Professor at the University of Toronto. We have three children of about your age and we are all looking forward to seeing you soon.

With best wishes, Yours sincerely Bruno and Hertha Mendel

(My transcription from the original letter in Eisinger 88).
Oben: Der Brief in dem Hertha Mendel mir und Walter Kohn mitteilt, ihre Familie möchte uns sponsern und uns einladen, bei ihnen $\mathrm{zu}$ wohnen.

DOEW 128
Toronto, I8 octobre '4I Arrivée le 22 octobre I94I

Cher M. Kohn et cher M. Eisinger,

J'espère que cela ne vous dérangera pas que nous vous écrivions tous les deux et non séparément. Nous avons entendu dire par Charles Kohn que vous êtes de bons amis. Nous voulons vous dire que nous aimerions devenir vos parrains et espérons vous avoir parmi nous très bientôt.

Je suppose que vous aimerez en savoir un peu sur nous. Nous sommes des réfugiés allemands qui ont quitté l'Allemagne en 1922 et qui sommes maintenant Canadiens. Mon mari est physiologiste et professeur à l'Université de Toronto. Nous avons trois enfants d'à peu près votre âge, et nous avons tous hâte de vous rencontrer bientôt.

Meilleurs vœux, Amicalement vôtre, Bruno et Hertha Mendel 
In September 1944, after I had returned to the Mendels from my summer job, Walter and I decided to make a serious attempt to volunteer for military service. We were rejected by the Royal Canadian Air Force because of our dubious nationality, but the $\mathrm{Ca}$ nadian Army accepted us and we were assigned the regimental numbers Bi64793 and B I64795, respectively. To my distress, I failed the vision test because of shortsightedness, but after using my glasses to memorize the eye chart, I asked to be tested again and this time, I passed without difficulty. Curiously, the notion that enlisting at the height of the war could lead to mutilation or death, did not seriously cross my mind. Such are the intimations of immortality of youth, besides, hadn't Papa survived his service in World War I? I do recall how proudly I wore my Canadian Army uniform as I walked down Yonge Street shortly after I was inducted-and was
Nachdem ich im

September 1944 von meinem Sommerjob zu den Mendels zurückgekehrt war, beschlossen Walter Kohn und ich, uns ernsthaft $z u$ bemühen als Freiwillige in den Militärdienst zu treten. Wir wurden von der Royal Canadian Air Force wegen unserer zweifelhaften Staatsbürgerschaft abgelehnt, aber die Canadian Army nahm uns auf und ich erhielt die Regiment-Nummer B I64795. Ich war betrübt, dass ich wegen meiner Kurzsichtigkeit am Augentest scheiterte, aber nachdem ich mit meiner Brille die Sehprobentafel auswendig gelernt hatte, bat ich noch einmal getestet $\mathrm{zu}$ werden, und diesmal bestand ich ohne Probleme. Seltsamerweise kam mir kein Gedanke, dass mein Eintritt ins Militär inmitten eines Krieges mich der Gefahr von Verstümmelung oder Tod aussetzen könnte: So mächtig ist die jugendliche Überzeugung der Unsterblichkeit - außerdem, hatte Papa nicht seinen Dienst im Ersten Welt-
À mon retour chez les Mendel, en septembre 1944 et à la fin de mon emploi d'été, Walter et moi prîmes la sérieuse décision de nous porter volontaires pour le service militaire. Au départ, l'Aviation royale canadienne rejeta notre candidature en raison de notre nationalité douteuse, mais au final, l'Armée canadienne nous accepta au sein de l'un de ses régiments et nous attribua les numéros de matricule suivants: Bi64793 et Bi64795. À mon grand désarroi, j'échouai à l'examen de la vue à cause de ma myopie. Après avoir mémorisé les caractères du tableau de l'optométriste à l'aide de mes lunettes, je demandai de repasser l'examen et cette fois-ci, je réussis sans difficulté. Il ne m'est, curieusement, jamais venu à l'esprit que je risquais de subir de graves blessures et risquais ma vie en m'enrôlant alors que la guerre était à son plus fort. Tel est le sentiment d'immortalité qui vient de pair avec la jeunesse! De plus, papa n'avait-il pas survécu à son ser- 
snapped by a sidewalk photographer.

Eisinger 100 krieg überlebt? Ich erinnere mich, wie stolz ich den Kampfanzug trug, als ich die Yonge Street entlangging, kurz nachdem ich eingerückt war - und ich von einem Fotografen am Gehsteig aufgenommen wurde.

DOEW 144-145 vice militaire pendant la Première Guerre mondiale? Je me souviens de la fierté avec laquelle je portais mon uniforme de l'Armée canadienne alors que je marchais sur la rue Yonge peu de temps après mon entrée dans les forces, et qu'un photographe qui passait par là me prit en photo.

Ma traduction

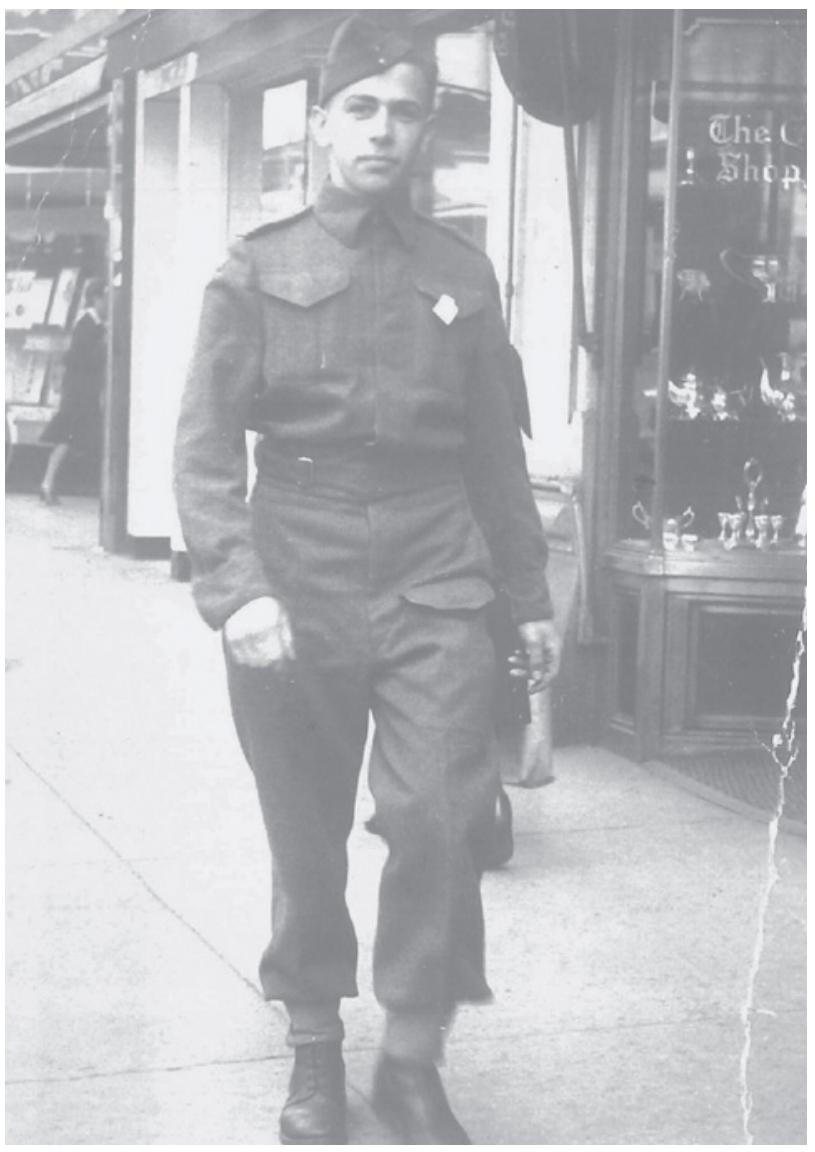


We were given a final 48-hour leave before our company was shipped overseas. Back in Camp Borden, we were about to climb into the waiting trucks with our kitbag, pack and rifle, when Walter and I were ordered to report to the company commander who informed us that we were to remain behind because we had been designated as 'sensitive' in case of capture.

Eisinger 103
Wir erhielten einen letzten 48-Stunden-Urlaub, bevor unsere Kompanie nach Übersee geschickt würde. Zurück im Camp Borden wollten wir gerade mit unserem Seesack, Rucksack und Gewehr in die wartenden Lastautos einsteigen, als Walter und ich vom Kompaniekommandanten den Befehl erhielten zu bleiben, offenbar, da wir im Falle einer Gefangennahme als jüdische Flüchtlinge besonders gefährdet gewesen wären.

DOEW 148-149
On nous accorda une dernière sortie de 48 heures avant que nous soyons déployés outremer. De retour à la base de Borden, nous étions sur le point de monter dans les véhicules de transport avec notre arme et notre équipement militaire quand Walter et moi fûmes convoqués par le commandant de la compagnie. Ce dernier nous informa du fait que nous ne pouvions pas être déployés comme les autres puisque nous étions 'à risque' en cas de capture.

Ma traduction
Upon graduating the School of Instruction I was promoted to the rank of corporal [...]

For the remainder of the war I trained a succession of platoons in the essential infantry skills, ranging from marksmanship and tank recognition to bayonet fighting. I did not take my responsibility lightly because after completing the course, my
Nach dem Abschluss der Ausbildungsschule wurde ich zum Unteroffizier befördert [...]

Bis zum Ende des Krieges bildete ich eine Reihe von Zügen in den Grundkenntnissen der Infanterie aus, von Schießkunst und Panzererkennung bis zum Bajonettkampf. Ich nahm meine Verantwortung ernst, da meine Schützlinge gleich nach
Après avoir été diplômé de l'École d'Instruction, je fus promu au grade de caporal [...]

Jusqu'à la fin de la guerre, je fus mis en charge de former un bon nombre de pelotons et de leur enseigner entre autres, à tirer avec précision, à reconnaitre les différents véhicules de combat et à se battre avec leur baïonnette lors de combat rapproché. 
charges were shipped straight to the European front.

Eisinger 104
Absolvierung des Kurses an die europäische Front verschifft wurden.

DOEW 149
Je ne prenais pas cette responsabilité à la légère puisque dès que le cours était complété, mes étudiants étaient directement envoyés au front européen.

Ma traduction

I felt set free once again, this time to return to the familiar and congenial student life I had left behind. And while I savored freedom of action and the privacy of civilian life, it does not surprise me that many veterans are nostalgic for their military service. [...] For my part, army service gave me the opportunity of meeting Canadians of many walks of life. The ease with which I had been accepted raised my confidence and made me feel even more at home in Canada.

Eisinger 105-106
Ich fühlte mich wieder frei, diesmal um zu dem bekannten und angenehmen Studentenleben zurückzukehren. Obwohl ich die Handlungsfreiheit und die Privatsphäre des zivilen Lebens schätze, verstehe ich, dass viele Veteranen nostalgisch an ihren Militärdienst denken. [...] Mir gab das Militärleben Gelegenheit, Kanadier aus vielen Schichten kennenzulernen. Die Ungezwungenheit, mit der ich aufgenommen worden war, hatte mein Selbstvertrauen gestärkt und mich in Kanada noch heimischer fühlen lassen.

DOEW 151
Je me sentis à nouveau libre lorsque je retournai à ma vie d'étudiant, qui était pour moi synonyme d'une vie familiale et agréable. Et même si j'ai savouré cette liberté retrouvée et l'intimité reliée à la vie civile, je ne suis pas surpris que de nombreux anciens combattants soient nostalgiques de leur service militaire. [...] Pour ma part, la vie militaire m'a donné l'occasion de rencontrer des Canadiens provenant de différents horizons. Le fait qu'on m'ait accepté si facilement renforça ma confiance en moi ainsi que mon sentiment d'appartenance au Canada.

Ma traduction 